\title{
Epigenetic correlation of interleukin expression between cigarette smoking and the therapeutic efficiency of periodontitis
}

\author{
H.-M. Chang ${ }^{1 *}$, T.-Y. Renn², N.-C. Teng ${ }^{3}$, Y.-K. Huang ${ }^{4}$ \\ ${ }^{1}$ Department of Anatomy and Cell Biology, College of Medicine, Taipei Medical University, Taipei, Taiwan \\ ${ }^{2}$ Graduate Institute of Medical Sciences, College of Medicine, Taipei Medical University, Taipei, Taiwan \\ ${ }^{3}$ School of Dentistry, College of Oral Medicine, Taipei Medical University, Taipei, Taiwan \\ ${ }^{4}$ School of Oral Hygiene, College of Oral Medicine, Taipei Medical University, Taipei, Taiwan \\ *e-mail: taiwanzoo@gmail.com
}

Key words: biomedicine, epigenetics, methylation, periodontitis, interleukin

Cigarette smoking is widely considered as the most important environmental risk factor for the development and progression of periodontitis (PD). Epigenetic modulation caused by cigarette smoking may serve as the underlying mechanism for the increased susceptibility of PD. However, although the impact of cigarette smoking on exaggerating the severity of PD has been well-documented, the condition that why patients with the same clinical phenotype responded differently to therapeutic efficiency has still largely unknown. Concerning interleukins (ILs) is the major cytokine participated in the modulation and progression of numerous inflammatory diseases; the present study is aimed to determine whether cigarette smoking would alter the methylation status of ILs, and therefore, contribute to the therapeutic discrepancy following PD. A total of 167 patients consisting of 79 males and 88 females were recruited in this study. Well-trained interviewers carried out the standardized personal interviews based on a structured questionnaire. Both the saliva samples and the clinical measurements [including the plaque index (Pi), bleeding on probing (Bop), and pocket depth (Pd)] were taken at the baseline and after the treatment of PD. The genomic DNA isolated from the gingival tissues was modified firstly by sodium bisulfite and then analyzed for DNA methylation levels of IL-1 $\beta$, IL-6, and IL-8 genes with direct sequencing. The levels of the cytokines were further determined by the enzyme-linked immunosorbent assay. The results indicated that patients suffered from PD with smoking behavior exhibited a higher percentage of hypomethylation of IL-1b gene and expressed high levels of IL-1 $\beta$, IL-6, and IL-8 than that of non-smokers. Moreover, the degree of Pi, Bop, and Pd after treatment was more evident in smoking patients, suggesting that the therapeutic efficiency of PD was negatively correlated with the extent of epigenetic change. As changes in methylation profile and subsequent increase in the expression of IL may be causally related to the poor response of treatment following PD, our findings thus not only provide a useful strategy for easy identification of patients at risk for poor therapeutic efficiency, but also helps to reduce the treatment costs that form the health economic perspective for the society. 